\title{
Measuring the Frailty Index of Multiple Myeloma Cancer Patients
}

\author{
Andrea Corradini \\ Design School Kolding \\ Aagade 10, 6000 Kolding, \\ Denmark \\ andrea@dskd.dk \\ Martin Bøgelund Hansen \\ IT University of Copenhagen \\ Rued Langgaards Vej 7, 2300 \\ Copenhagen, Denmark \\ marha@itu.dk \\ Toma Savic \\ University of Southern Denmark \\ Universitetsparken 1, 6000 \\ Kolding, Denmark \\ tosav@sdu.dk
}

Permission to make digital or hard copies of all or part of this work for personal or classroom use is granted without fee provided that copies are not made or distributed for profit or commercial advantage and that copies bear this notice and the full citation on the first page. To copy otherwise, to republish, to post on servers or to redistribute to lists,

requires prior specific permission and/or a fee.

PervasiveHealth 2016, May 16-19, Cancun, Mexico

Copyright @ 2016 EAI 978-1-63190-051-8

\begin{abstract}
We report on a responsive web-based application that we have been developing for the cancer hospital in Vejle, Denmark. The application administers and handles systematic frailty scoring of patients with multiple myeloma and helps doctors make a more efficient and effective treatment choice. The application is currently being tested with a small number of patients and is to replace the frailty measurement system used until now, which is done by the doctor on a per patient basis.
\end{abstract}

\section{Author Keywords}

Web application; mobile health; frailty scoring.

\section{ACM Classification Keywords}

H.1.2. User/Machine Systems: Human information processing; H.4.1. Types of systems: Decision support (e.g., MIS); H.5.3 Group and Organization Interfaces:

Web-based interaction.

\section{Introduction}

Over the last years, cancer therapy has improved and become more complex. Beside surgery, chemotherapy, radiotherapy, and hormonal therapy, newer targeted treatments are now available. While standardized, most of these antineoplastic treatments are still potentially toxic. In Denmark, each type of cancer requires a particular combination of treatments and protocols that 


\section{Our Application and the Patient's Electronic Health Record (EHR)}

The hospital in Vejle uses Cambio COSMIC as EHR

(http://www.cambiohealthcar e.co.uk/Cosmic/). It only allows including certain file

types to each patient's record. Because of that, instead of running our application from inside COSMIC, doctors use a standalone version of it. After determining the frailty score for a patient (the patient is validated using its identification number, called CPR number), a report in PDF format is created and then pipelined to an externa application, called onBase

that makes it possible to add documents to the EHR. When a doctor wishes to look up a patient's frailty score, s/he can access the patient's EHR and consult the report saved into it. Figure 1 shows a diagram with the steps necessary to include the data calculated with the frailty application into the EHR. are defined in what are called integrated cancer pathways ${ }^{1}$ (or "kræftpakker" in Danish). Such standardization does not always apply to older patients such as those suffering from multiple myeloma. Older patients are less likely to receive all types of standard cancer therapies compared to younger individuals. Each therapy requires an adaption to reduce the risk of toxicities, especially when a sequential combination of therapies is chosen, for each of them add cumulative toxicities. Several non-cancer related factors influence the process of decision making. While chronological age per se is not sufficient to determine tolerance to cancer treatment, elderly patients usually suffer from multiple co-morbidities, have disabilities, cognitive impairments, depression, mobility impairment, malnutrition, polypharmacy, and/or live in a difficult psychosocial context. Thus, beside usual cancer-related factors such as tumor histology, site, extension, etc. the treatment decision for these patients, needs to be made in collaboration with the patient's values, preferences and based on his/her functional status. Comorbidity and the aging process can limit the capability of a patient to tolerate a certain therapy $[3,7]$

In order to find the right balance between positive effects and malignant side effects on the patient's health status, we base the assessment of multiple myeloma cancer patients on a set of frailty markers. Frailty represents a state of reduced resistance to stress that can ultimately lead to increased vulnerability and risk of adverse outcomes such as death. The manifestations of frailty include weight loss, exhaustion, weakness, decrease in physical activity,

1 https://sundhedsstyrelsen.dk/en/disease-andtreatment/cancer/cancer-pathways. and so on, and represent anything that can interfere with the functional status of a patient [1]

A few studies show that older cancer patients have a high prevalence of frailty markers and some of them are predictive to cancer treatment toxicities $[6,10]$. Therefore, in older cancer patients, the concept of frailty may be a useful approach to detect vulnerability and then base the treatment decisions on that. A frailty score can help modulate the proposed therapy while at the same time to minimize or to prevent complications. Our application categorizes patients as frail, unfit, or fit based on a numeric score calculated after collecting patient's information from a conglomerate of published and validated methods.

\section{The Frailty Application}

The creation of our application was influenced by the web-based application for desktop computers proposed in $[8,9]$. In that system, the patients only go through the scoring once throughout the entire treatment process, notably at the time of diagnosis. We make it possible to have repeated assessments for the same patient at different times throughout the treatment process. This makes it possible to adjust the measures that were initially set during the treatment process and accordingly use the new assessment to optimize the rehabilitative and palliative measures. Our application allows for several scoring at different times during the patient's journey at the hospital. The first frailty scoring is carried out at the time of diagnosis, the second after three months and the final, third one, when the first line of treatment is completed or at the latest, after one 
year of treatment. We also expanded the frailty scoring system in order to accommodate the registration of parameters that are particularly relevant for palliation and rehabilitation. Moreover, our application can be integrated with the electronic health record (Figure 1), it is web-based i.e. it runs smoothly on any operating systems and it is responsive meaning that it runs on any device, from desktop computers to smartphones (Figure 2).

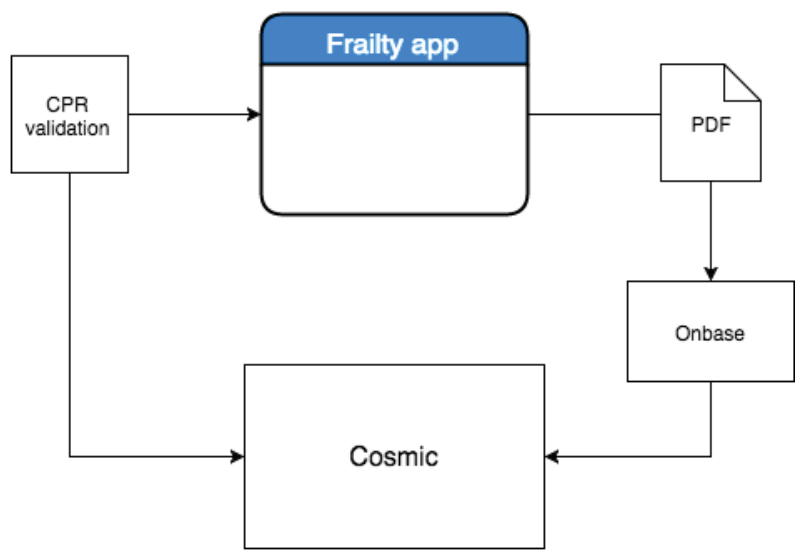

Figure 1: Diagram of the overall system architecture and the process to include the frailty score into the EHR.

Nurses and doctors input a set of parameters for each patient, notably, the values of two standard assessments of quality of life EORTC QLQ-C15 [11] and EORTC QLQ-C30 [12], the questionnaires on activities of daily living (ADL) [4], the instrumental activities of daily living assessment (IADL) [5], and the Charlston's comorbidity index (CCI) [2]. By a weighted combination of these values, the application calculates the patient's frailty score and helps doctors identifying the most vulnerable patients and subsequently to target individual interventions and therapy. Since many factors can interfere with the results of the assessment, each patient is evaluated at different times. In this way, the doctor has a better overview of the impact of the cancer on the patient's health at the time of each assessment. For instance in case of an aggressive or advanced cancer, a patient may be erroneously classified as frail but should not be refused an aggressive treatment on the basis of it. In fact, the erroneous frailty assessment can change with optimal cancer therapy.

We conducted a preliminary test with two patients, one nurse and one medical doctor. When asked about the project, the patients stated that "...it makes perfect sense.." and could be useful for their treatment. This initial test phase showed also that the patients do not have yet an attitude towards it. They preferred the nurse to collect the data, ask questions and enter the answers in the system instead of doing so themselves. The nurse and the doctor are instead very motivated and keen in using the application. According to the nurse, the application ".. can help nurses prepare and make every consultation more individual and useful since it will help us see the patients development.."

\section{Conclusions}

The optimal management of elderly multiple myeloma patients is challenging. A correct estimation of the patient's vulnerability can guide the decision making process regarding the appropriate antineoplastic treatment. We developed a web application that performs all the functions that the hospital is doing physically, as well as new features that give also the patients the opportunity to fill out a part of their own frailty assessment. The application combines several 

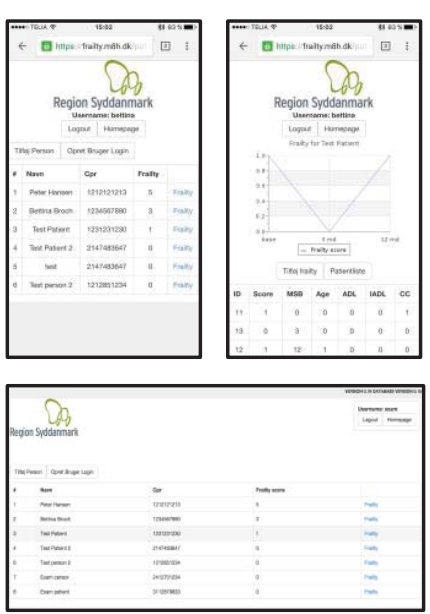

Figure 2: The user interface of the application as it looks like on a mobile phone (above) and on a desktop computer (below), respectively. technical improvements over similar applications, especially an individual parametrization for each patient and a responsive interface, i.e. the support of various screen sizes and haptic input. It performs all functions in an elegant and functional way that is user-friendly, safe, secure and easy to further develop. From preliminary testing carried out on a daily basis in a highly sensitive environment like a hospital, the

\section{References}

1. Baijal, P., and Periyakoil, V. 2014. Understanding frailty in cancer patients. Cancer J. 20(5):358-66.

2. Charlson, M.E., Pompei, P., Ales, K.L., and MacKenzie, C.R. 1987. A new method of classifying prognostic comorbidity in longitudinal studies: Development and validation. Journal of Chronic Diseases. 40(5):373-83.

3. Hurria, A. 2013. Management of elderly patients with cancer. Journal of the National Comprehensive Cancer Network. 11(5):698-701.

4. Katz, S., Ford, A.B., Jackson, B.A., and Jaffe, M.W. 1963. Studies of illness in the aged. The index of ADL: a standardized measure of biological and psychosocial function. JAMA. 185(12):914-919.

5. Lawton, M.P., and Brody, E.M. 1969. Assessment of older people. Self-maintaining and instrumental activities of daily living. The Gerontologist. 9(3), 179-186.

6. Makary, M.A., Segev, D.L., Pronovost, P.J., Syin, D., Bandeen-Roche, K., Patel, P., Takenaga, R., Devgan, L., Holzmueller, C.G., Tian, J., and Fried, L.P. 2010. Frailty as a predictor of surgical outcomes in older patients. Journal of the American College of Surgeons. 210(6):901-908. application turned out to be robust and reliable. More testing is however necessary to help us further

developing the application so that it can be tailored to older cancer patients.

\section{Acknowledgements}

Our thanks to Udviklingsrådet of Region Syddanmark

for funding this research and development project.

7. Pal, S.K., Katheria, V., Hurria, A. 2010. Evaluating the older patient with cancer: understanding frailty and the geriatric assessment. CA: A Cancer Journal for Clinicians. 60(2):120-132.

8. Palumbo, A. et al. 2011. Personalized therapy in multiple myeloma according to patient age and vulnerability: a report of the European Myeloma Network (EMN). Blood J. 118(17):4519-4529.

9. Palumbo, A. Geriatric assessment predicts survival and risk of serious adverse events for elderly newly diagnosed multiple myeloma patients: a report of International Myeloma Working Group.

10. Puts, M.T., Hardt, J., Monette, J., Girre, V., Springall, E., and Alibhai, S.M. 2012. Use of geriatric assessment for older adults in the oncology setting: a systematic review. Journal of the National Cancer Institute. 104(15):1133-63.

11. QL Coordinator Quality of Life Unit, European Organisation for Research and Treatment of Cancer (EORTC) Data Center, EORTC QLQ-C15 QL, 2005.

12. QL Coordinator Quality of Life Unit, European Organisation for Research and Treatment of Cancer (EORTC) Data Center, EORTC QLQ-C30 Scoring manual. 3rd edition, 2001. 